\title{
Pathway Enrichment Based on Text Mining and Its Validation on Carotenoid and Vitamin A Metabolism
}

\author{
Andra Waagmeester, ${ }^{1,2,3}$ Piotr Pezik, Susan Coort, ${ }^{3}$ Franck Tourniaire, ${ }^{4}$ \\ Chris Evelo, ${ }^{3}$ and Dietrich Rebholz-Schuhmann ${ }^{1}$
}

\begin{abstract}
Carotenoid metabolism is relevant to the prevention of various diseases. Although the main actors in this metabolic pathway are known, our understanding of the pathway is still incomplete. The information on the carotenoids is scattered in the large and growing body of scientific literature. We designed a text-mining work flow to enrich existing pathways. It has been validated on the vitamin A pathway, which is a well-studied part of the carotenoid metabolism. In this study we used the vitamin A metabolism pathway as it has been described by an expert team on carotenoid metabolism from the European network of excellence in Nutrigenomics (NuGO). This work flow uses an initial set of publications cited in a review paper (1,191 publications), enlarges this corpus with Medline abstracts (13,579 documents), and then extracts the key terminology from all relevant publications. Domain experts validated the intermediate and final results of our text-mining work flow. With our approach we were able to enrich the pathway representing vitamin A metabolism. We found 37 new and relevant terms from a total of 89,086 terms, which have been qualified for inclusion in the analyzed pathway. These 37 terms have been assessed manually and as a result 13 new terms were then added as entities to the pathway. Another 14 entities belonged to other pathways, which could form the link of these pathways with the vitamin A pathway. The remaining 10 terms were classified as biomarkers or nutrients. Automatic literature analysis improves the enrichment of pathways with entities already described in the scientific literature.
\end{abstract}

\section{Introduction}

C AROTENOIDS ARE NATURAL nutritional compounds. Fruits, vegetables and derived products constitute the major sources of these lipid-soluble pigments. There is strong evidence that high dietary intake of these compounds is beneficial for humans, and that it is associated with a lower risk of developing cardiovascular diseases, cancers, and agerelated eye diseases (Amiot-Carlin, 2008; Bendich and Olson, 1989; Krinsky, 1993). Several mechanisms have been described that explain at least in part the observed effects. Examples include: (1) a role in the scavenging of free radicals, (2) the enhancement of gap-junction communication, (3) a role in immunomodulation, (4) reduction of the risk of site-specific cancer and heart disease, and (5) the protection of eye tissue (Yeum and Russell, 2002). Among the more than 600 identified carotenoids, around 10\% are categorized as pro-vitamin A, meaning that they can be cleaved by the organism to produce retinal, the precursor of vitamin A (retinol). Harrison (2005) estimated in 2005 that more than 100 million children worldwide suffer from vitamin A deficiency, and indicated that an increased understanding of the metabolic processes concerning the absorption of carotenoids could lead to better nutrition and thereby in general to an increase in the health of several populations. Getting a profound understanding of the metabolic pathway of carotenoid metabolism is assisted by the integration of a variety of study results from different sources and biomedical research domains (Voutilainen et al., 2006).

Pathways are abstract and functional representations of biological knowledge (Cary et al., 2005). Our understanding of their functioning is essential in the analysis of genomic results (Dahlquist et al., 2002) and, because such an approach requires accurate and up-to-date representations of pathways, this research work is ongoing. For the creation of such representations the scientific curators (biologists and other

\footnotetext{
${ }^{1}$ European Bioinformatics Institute, Hinxton, Cambridge, CB10 1SD United Kingdom.

${ }^{2}$ Maastricht ICT Competence Centre, Maastricht University, Maastricht, The Netherlands.

${ }^{3}$ Department of Bioinformatics, BiGCaT, Maastricht University, Maastricht, The Netherlands.

${ }^{4}$ Human Nutrition Research Centre, University of Newcastle upon Tyne, Faculty of SAgE, Newcastle upon Tyne, United Kingdom.
} 
domain experts) merge their expert knowledge with information from biological databases (Bader et al., 2006), and available scientific literature.

In biology, the Medline database is the primary resource of scientific literature. It contains more then 18 million references to scientific journal publications in the biomedical field, including author information and abstracts. The rate of growth of Medline is so high that scientists have big difficulties to keep up to date (Jensen et al., 2006). New approaches to filter information from the scientific literature are required. Traditional procedures for gathering documents from a corpus (i.e., a collection of documents. PubMed could be considered as a corpus of biomedical abstracts) rely on selecting a set of appropriate key words with which one hopes to retrieve as many relevant publications as possible from the document collection at hand. Measures have been defined to monitor and optimize the retrieval for a given task to the selection of relevant documents (Jensen et al., 2006). Whenever the resulting set of references is too overwhelming, one coping strategy is to limit the search to review articles. To this end, PubMed, the Web-based interface to Medline, offers functionality to limit the searches to retrieve only reviews. By manually selecting appropriate reviews scientists acquire references to other relevant articles.

A literature search is optimal if both precision and recall are high. Precision is the number of relevant documents in all retrieved documents. Recall is the portion of retrieved documents out of all relevant documents in the corpus (Shatkay and Feldman, 2003). In other words, the recall is the ratio between the true positives and the sum of the true positives and the false negatives. The precision is the ratio between the true positives and the sum of the true positives and the false positives. A literature search is optimal if the results are generated with high recall and high precision at the same time, which is difficult to achieve becvause often high precision hinders high recall and vice versa (see the explanations below).

Two factors have a strong influence on recall and precision. The first factor is the polysemy (a term denoting multiple meanings) of key words used in user queries leading to their ambiguous interpretation in the text. Highly ambiguous terms decrease precision, because the number of false positives could increase, that is, the more senses a term has the more diverse the final retrieval of documents (e.g., the term cancer denoting a disease and a species). The second factor is synonymy (the use of different terms to denote the same concept). If a concept is represented by more then one term, and neither the query system nor the user formulating the query considers all relevant synonyms to generate a complete query, this changes the recall due to the potential increase of false negatives. An example that illustrates the influence of the latter factor is the retrieval of documents describing MRI (magnetic resonance imaging) (Spasic et al., 2008). This imaging method is widely used in medicine. The same underlying physical method is used in chemistry for spectral analyses, but is here called NMR (nuclear magnetic resonance). Documents describing NMR could be relevant for someone interested in MRI. Unawareness of synonymous terms leads to missing relevant literature. PubMed uses thesauri like MESH to overcome some of these problems. The link between NMR and MRI will be found in MESH. But synonymy could be so subtle that it would require very big thesauri, and then again the excessive use of synonyms creates the risk of increasing ambiguity. Because of these limitations in the use of key words as query terms and due to the increasing volume of scientific literature, we are prone to miss relevant literature.

Biological network and pathway models are needed to integrate different types of genomic results in a systems biology approach to understand for instance micronutrient health (van Ommen et al., 2008). This approach is currently limited by the quality of the available biological pathways. To improve this, curators integrate knowledge from various scientific domains. Bercause each domain has specific sets of journals, this means that many journals need to be explored. So in pathway curation the chance of missing important information is thus likewise increased. What we present here is an integrated approach where the pathway content is used to start a text-mining effort, and the results are represented in such a way that they can easily be integrated into the pathway itself by manual curation. In our study we have compiled a text-mining work flow for finding potential pathway entities in the literature for a given context (using Vitamin A and carotenoids as an example). Other researchers have used text mining to built abstract representations of relations in biology. Spasic et al. (2008) have used text-mining solutions to generate a controlled vocabulary of terms, which is a set of relevant words or phrases. In a different study, the text-mining solution generated networks of related concepts (Chen and Sharp, 2004). Our approach is similar to those mentioned above, but differs in the sense that we go one step further. Through a combined representation of the existing pathway and the newly found terms we allow people knowledgeable in the domain under scrutiny to combine already formulated pathway knowledge with textmining results. Thus, offering an integration of automated and manual knowledge collection. In our study, we assessed the relevance of the identified terms in collaboration with domain experts and integrated a number of candidates into the latest pathway representation.

In the present study we apply a semiautomatic approach to identify pathway entities in Medline abstracts. We started with a pathway developed for vitamin A metabolism, which is the best-understood subpathway of the carotenoid pathway (Fig. 1). The text-mining approach was initiated from a single review article with 1,191 references (Balmer and Blomhoff, 2002). In the first step a corpus of Medline abstracts was automatically generated based on these references. This corpus was subsequently analyzed for pathway-related terms. The final output is a ranked list of terms that have been validated by curators for their relevance and novelty to the pathway under scrutiny. Our approach improves the process of generating, enriching and updating pathways.

\section{Methods}

Our text-mining solution makes use of the text-mining infrastructure at the European Bioinformatics Institute (EBI) where individual modules solve specific tasks (Whatizit, MedEvi) (Kirsch et al., 2006; Rebholz-Schuhmann et al., 2008). In addition to filtering out pieces of information the solutions also provide explicit links from the identified information to entries in biomedical data resources such as Uniprot (Bairoch et al., 2005), ChEBI (Degtyarenko et al., 2008), and the Human Metabolome Database (Wishart et al., 2007). Uniprot or the Universal Protein Resource, came into existence by merging 


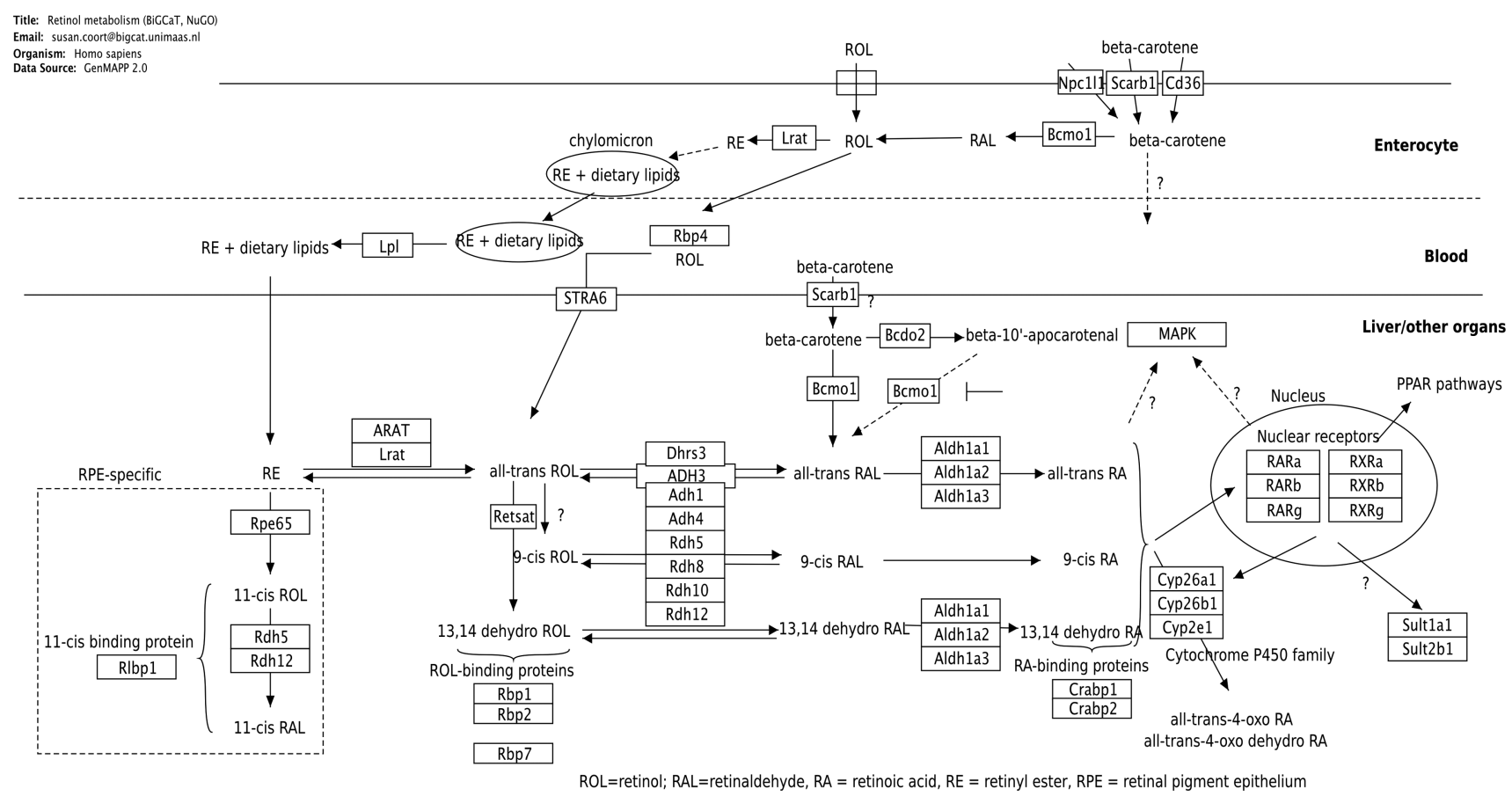

FIG. 1. The metabolic pathway of vitamin A (retinol) metabolism constructed by a NuGO focus team on carotenoid metabolism (source: http://www.wikipathways.org November 2007).

Swiss-Prot, TrEMBL, and PIR. ChEBI is database capturing chemical entities active in a biological context, and the Human Metabolome Database cotains information of Human endogenous metabolites.

\section{Corpus generation}

In the first step we generated a seed corpus (SC) from the comprehensive review publication by Balmer and Bloomhoff (2002) that provides 1,191 references (see Fig. 2A). Then we generated a second corpus consisting of a large set of abstracts from Pubmed by using the query "carotenoids" (called "query selected corpus," QSC, see Fig. 2B). Each abstract from the QSC is assessed for the relevance to our topic by measuring the similarity to the SC (see Fig. 2). The goal was to generate a large set of documents from the scientific literature that contains all relevant documents for the topic, that is, the carotenoid pathway, without imposing any bias on the selection. To this end the documents from the QSC are assessed against the documents from the SC to generate the "similarity extended corpus" (SEC, see Fig. 2C).

\section{Relevance assessment of the QSC documents for the $S C$}

For the SC all the titles and abstract texts were combined and processed together, whereas the documents from the QSC were processed individually. First, the sentences in the texts were marked up explicitly, and the part-of-speech information was added to the lemmatized tokens, that is, tokens represented in their dictionary form. Every document was transformed into a vector of its lemmatized tokens after removing noninformative tokens provided from a stop list, and the frequency of the lemmatized tokens in the document was kept to rank the tokens.
For every document we selected the most discriminative 20 terms according to the following procedure:

1. For each lemmatized term found in a document Dunning's log-likelihood value has been computed. Typically, likelihood values are calculated in the context of hypothesis testing, which follows a binomial distribution; that is, true or false. The values are used to either accept or reject a null-hypothesis. Dunning et al. adapted the log likelihood ratio test to be applicable in a set with multinomial distributions. This adaptation makes the log-likelihood ratio test applicable in automatic text analysis. When counting word occurrences in a document the resulting set follows a multinomial distribution. Dunning's log likelihood value gives an indication if a term is informative in a given context.

2. All terms are ranked according to their log-likelihood values where the highest log-likelihood value obtained is ranked at 1 . The ranks are then used to compute the term weight according to the following formula: the inverse of $[1+\log (\mathrm{rank})]$. This transformation results in a smoother distribution of term weights for a given document.

3. The same procedure is applied to generate the term vector of the SC. Because the SC of the carotenoid pathway contains 1,191 documents, a much larger vector containing the weights of 1,000 terms was used.

4. For each document from the QSC the cosine similarity score is computed between the document's vector and the vector of the SC. Notice that the term weight can be equal to 0 whenever a term that is contained in the SC is not found in the document vector being tested for similarity. The documents from the QSC are ranked according to their decreasing cosine similarity, which indicates their relevance. The best relevant documents are added to the final SEC. 
A

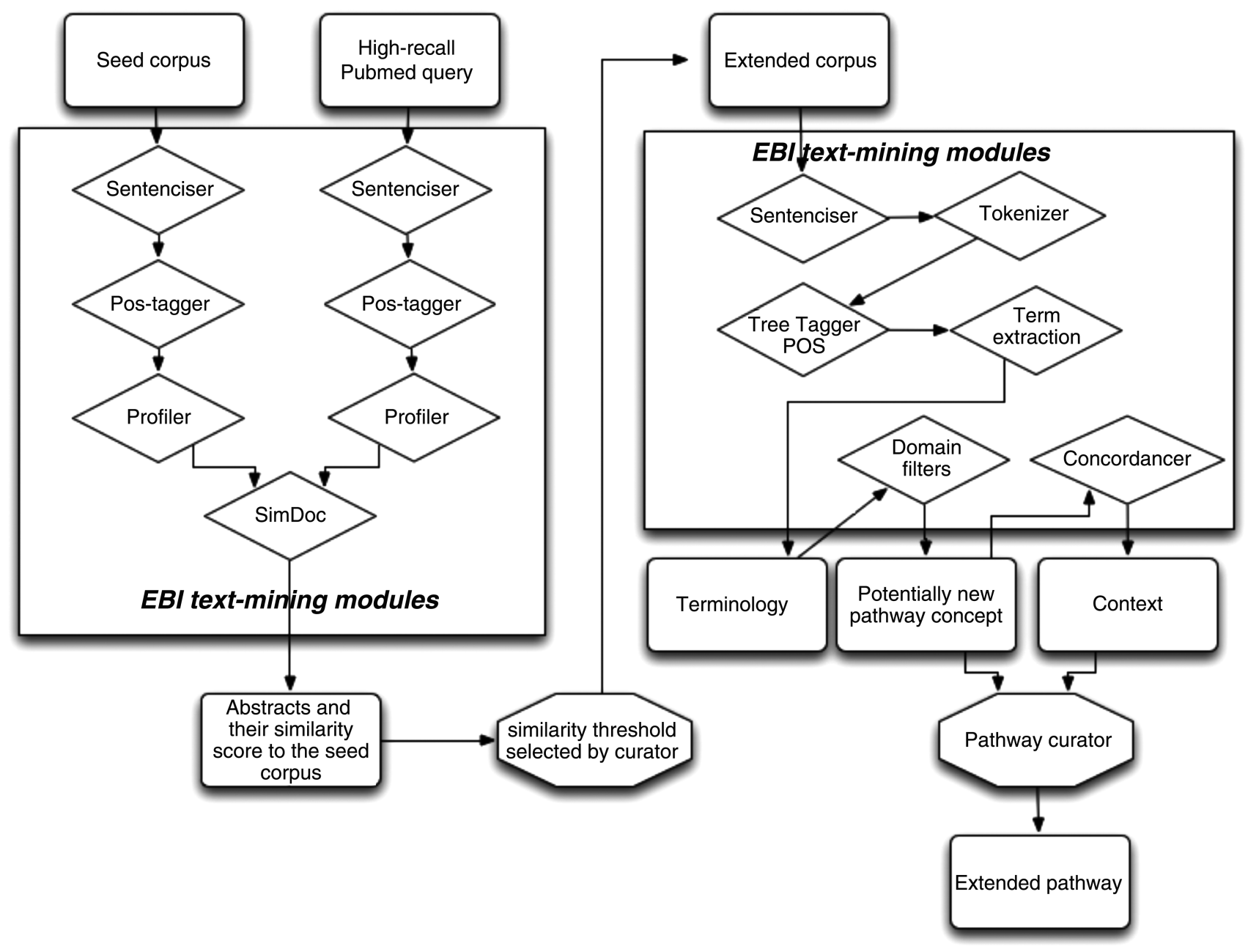

FIG. 2. A two-step text-mining work flow for the enrichment of pathways. During the first phase a corpus of manually assessed abstracts $(\mathbf{A})$ is extended in a semiautomatic manner with abstracts obtained from a high-recall query from PubMed (B). This is extended corpus (C) is then input to the second phase in which terms are extracted. The resulting terminology comprises potential pathway concept, which is available to pathway curators to extend a pathway.

5. A random set of abstracts from the QSC ranked according to their similarity score to the SC was evaluated for their relevance to the studied topic. Based on this assessment we derived a threshold for the similarity score. The selection of the threshold for similarity will be explained in detail in the results section. All abstracts with a similarity score higher than the selected threshold were added to the final SEC.

\section{Extracting terminologies containing potential pathway entities}

In the next step the documents from the SEC were processed (see Fig. 2C) to deliver a list of statistically significant terms, which are then filtered on relevance to the curated pathway. Potentially novel pathway entities are assumed to belong to the following semantic types: (1) proteins, (2) genes, (3) enzymes, (4) chemical compounds representing metabolites, that is, chemicals being intermediate substances in metabolic processes, and (5) other chemical compounds. Our analysis was tuned to identify multiword terms in the SEC, because we applied part-of-speech patterns and the mutual information test that measures the association strength between the constituents of multiword terms (Oakes, 1998). Recognition of multiword terms in natural language is complex when the words are not consecutive in a sentence, and this may lead to identification of shorter terms as subconcepts. Presentation of results containing such subconcept to an expert is often enough to make him see the larger picture.

In addition, we estimated the usage patterns for each term from the SEC, such as the evenness of a term's distribution in comparison to its relative frequency in the corpus.

Evenness is measured with the Juilland Dispersion (JD). To this end the corpus is separated into 100 segments, that is, bins containing similar numbers of sentences. Then we compute the frequencies $\left(X_{-} t\right)$ of all statistically identified terms in all segments and determined the mean of their frequency over all segments $\left[X=\right.$ mean $\left.\left(X_{-} t\right)\right]$ and the standard deviation $\left[S=\right.$ standard deviation $\left.\left(X \_t, X\right)\right]$. The JD is calculated as follows: 


$$
J D=100^{*}\left(1-\frac{V}{\sqrt{n-1}},\right.
$$

where $(n=100)$ is the number of segments in the SEC and $V$ is the inverse of the mean of frequencies $(X)$ normalized by the standard deviation $(S)$ :

$$
V=\frac{S}{X}
$$

A high dispersion value $\mathrm{JD}(t)$ occurs if a term $t$ is frequent in almost all segments of the corpus. Comparing a term's relative frequency to its dispersion value could be a useful clue in the identification of potentially novel entities due to the following two interpretations:

1. If the term is frequent and has a high dispersion value, then it can be assumed to denote a concept, which is likely to be very important in the context of the pathway represented by the corpus. However, it is likely that such entities have already been included into the pathway representation, because they are mentioned consistently throughout the corpus, and thus were likely to also occur in the documents used to create the initial pathway.

2. If the term is frequent but has a low dispersion value, then this signifies that a term is local to a smaller number of segments of the corpus. Given that we only accept pathway-relevant named entities such as Uniprot entries, metabolites, and other chemical compounds as candidate pathway entity, a combination of high frequency and low dispersion could be indicative of a situation where the term is used in a few abstracts that report on important and new findings related to the pathway. Such entities are more likely to appear as new suggestions for pathway extension but still have to be evaluated for relevance.

\section{Selecting the SC}

In our efforts to develop a suitable text-mining approach for pathway generation we focused on an important part of the carotenoid metabolism, the vitamin A metabolism. The reason for focusing on this part is twofold. It is the bestexplored part of the carotenoid pathway, and thus serves as a good benchmark in comparison to less understood pathways, and in addition, the review article by Balmer and Bloomhoff (2002) formed an ideal SCon gene regulation by retinoic acid. This review was an important source of information for the pathway editors from $\mathrm{NuGO}$ in that it listed 1,191 articles reporting relevant information to the vitamin A pathway. $\mathrm{NuGO}$ is the European Network of Excellence in Nutrigenomics (see http://www.nugo.org).

\section{Increasing the recall}

We have used the abovementioned 1,191 references as a SC. We extended the SCto 13,579 Medline abstracts by setting the cutoff point for the similarity score at 0.07 . The similarity threshold was obtained as a result of an integrated process using the curation capabilities of domain experts and the text processing capabilities of a novel text mining solution. The three following steps were followed:

Step 1: we calculated the similarity score for an increasing number of potentially relevant abstracts (see Fig. 3). The different distributions in Figure 3 depict the similarity scores applied to term vectors of $30,100,500$, or 1,000 terms, respectively. The introduction of more terms as vector elements decreased the similarity score of initially

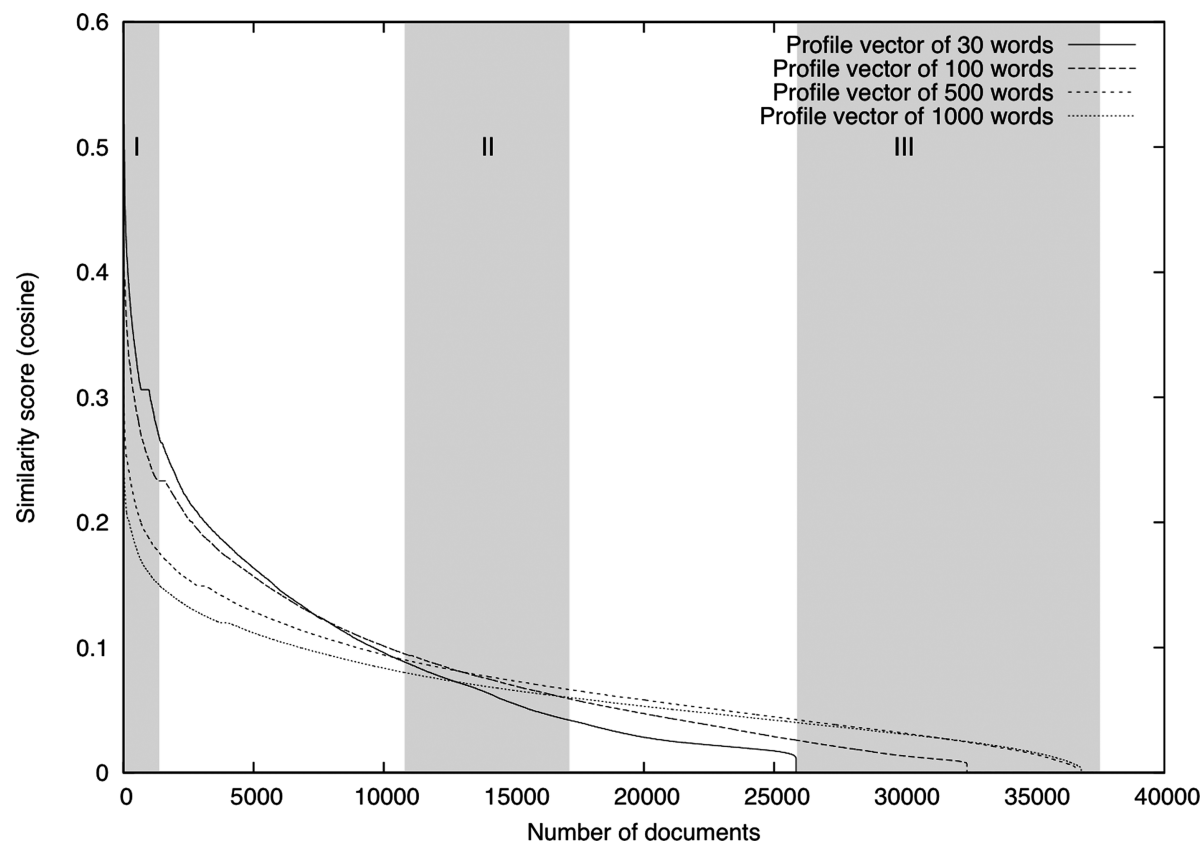

FIG. 3. Distribution of similarity scores between a corpus containing potentially relevant publications and a seed corpus. The different graphs depict the difference in distribution for different number of informative terms in simdoc vectors. 
better-ranked documents and increased the similarity score of documents at lower ranks. We conclude that the increase of the term vector size leads to an increase in noise (i.e., terms that are not specific to the domain). We select the cutoff point at the value where the increase of terms in the vector leads to a decrease of the ranking for relevant documents and vice versa. This cutoff point was found to be in the range of 15,000 and 20,000 documents with similarity scores between 0.06 and 0.08 .

Step 2: three groups of documents were selected that were all represented by term vectors with 1,000 entries (Fig. 3). The first section was picked from the part of the distribution where the term vectors showed high similarity scores (above 0.15 ). The second part was picked from the region where the different graphs intersect with similarity scores between 0.06 and 0.08 . The third part was selected from the tail of the graph, with similarity scores below 0.04. From the first group we selected 20 abstracts with a similarity score just above 0.15 . For the second group we selected 20 abstracts with a similarity score around the median value of 0.07 and for the last group 10 abstracts were selected with a similarity score below 0.04 and 10 with a similarity score around 0.01. This evaluation method is known as precision at rank (Trec, 2007).

Step 3: now, all the selected abstracts were presented to the domain experts from NuGO in random order. The abstracts were assessed for their relevance to the studied pathway and based on the evaluation by domain experts, the precision was calculated for each section (Table 1).

The precision in the first section was $100 \%, 60 \%$ in the second, and $15 \%$ in the last one (Table 1). This outcome confirms our expectation that the cutoff point should be selected in a range where the similarity score indicates that the term vectors mainly contain relevant terms. We decided to take the median value of the second section, which corresponds to a similarity score of 0.07 .

\section{Results}

The initial query result on the PubMed query "carotenoids" resulted in a set of 51,628 references of which 34,682 contained an abstract. Of these 34,682 documents 13,579 had a similarity score $>0.07$, and were considered to be relevant to the construction of the vitamin A metabolism pathway.

\section{Increasing journal coverage}

The corpus expansion from 1,129 abstracts to 13,579 abstracts led also to changes in the distribution of journals in the corpus (Fig. 4). By expanding the corpus we observed an increase in the contribution of distinct journals that were not listed in the SC. The extended corpus contained 3,307 distinct journals out of which 2,586 journals were not part of the seed corpus. This clearly shows that the automated corpus expansion has lead to larger journal coverage, which is practically not achievable by manual selection of scientific articles.

\section{Extracting the key terminology}

From the expanded corpus of 13,579 abstracts, we were able to extract 89,086 unique terms. A term may consist of
Table 1. Precision at Rank Evaluation

\begin{tabular}{lrcr}
\hline Rank & Pubmed Id & Similarity score & Relevant \\
\hline$A$ & & & \\
1141 & 7141883 & 0.154899 & + \\
1142 & 10693163 & 0.154814 & + \\
1143 & 1317113 & 0.154776 & + \\
1144 & 7486470 & 0.154721 & + \\
1145 & 8843984 & 0.154714 & + \\
1146 & 9795972 & 0.154656 & + \\
1147 & 438897 & 0.154653 & + \\
1148 & 8504149 & 0.154643 & + \\
1149 & 9212349 & 0.154601 & + \\
1150 & 15735074 & 0.154554 & + \\
1151 & 16614418 & 0.154553 & + \\
1152 & 7346779 & 0.154543 & + \\
1153 & 8823154 & 0.154535 & + \\
1154 & 9108943 & 0.154528 & + \\
1155 & 16365078 & 0.154524 & + \\
1156 & 3632216 & 0.154506 & + \\
1157 & 8503360 & 0.154503 & + \\
1158 & 17234732 & 0.154502 & + \\
1159 & 16177187 & 0.154477 & + \\
1160 & 10846237 & 0.154368 & \\
precision & $100 \%$ & & +
\end{tabular}

$\begin{array}{ll}0.060701 & + \\ 0.061302 & + \\ 0.065686 & + \\ 0.065687 & + \\ 0.065687 & + \\ 0.06569 & + \\ 0.065714 & + \\ 0.065715 & - \\ 0.083392 & - \\ 0.083394 & + \\ 0.083396 & - \\ 0.083397 & - \\ 0.08342 & - \\ 0.083421 & + \\ 0.083421 & + \\ 0.083425 & + \\ 0.083435 & - \\ 0.083444 & - \\ 0.083453 & + \\ 0.083459 & -\end{array}$

$\begin{array}{rl}12060479 & 0.060701 \\ 1309942 & 0.061302 \\ 9272132 & 0.065686 \\ 16060221 & 0.065687 \\ 16688767 & 0.065687 \\ 9173086 & 0.06569 \\ 17493127 & 0.065714 \\ 9720977 & 0.065715 \\ 1873990 & 0.083392 \\ 14692515 & 0.083394 \\ 16232082 & 0.083396 \\ 12671093 & 0.083397 \\ 3572247 & 0.08342 \\ 3107194 & 0.083421 \\ 1328400 & 0.083421 \\ 11978137 & 0.083425 \\ 10932161 & 0.083435 \\ 10080695 & 0.083444 \\ 10356420 & 0.083453 \\ 4424510 & 0.083459\end{array}$

$$
\begin{aligned}
& + \\
& + \\
& + \\
& + \\
& + \\
& + \\
& + \\
& - \\
& - \\
& + \\
& - \\
& - \\
& + \\
& + \\
& + \\
& - \\
& +
\end{aligned}
$$$$
\text { precision } \quad 60 \%
$$

C 
TABle 1. (Continued)

\begin{tabular}{lccc}
\hline Rank & Pubmed Id & Similarity score & Relevant \\
\hline 25002 & 7679173 & 0.041907 & - \\
25001 & 13679861 & 0.041911 & - \\
25000 & 2376553 & 0.041911 & - \\
precision & $15 \%$ & & \\
\hline
\end{tabular}

Three sections were picked with relatively high, intermediate, and low similarity scores. The precision was calculated for each section to obtain a threshold for relevance.

multiple words. This extracted terminology contains domain independent terms, because the extraction methods did not impose any domain-specific filter. Examples of domain independent terms are: "Etude du Vieillissement Art," "kg body wt," or "Southwest Oncology Group." This shows that not all statistically significant terms are relevant to the analyzed pathway even though they were extracted from a corpus of relevant abstracts.

From all extracted terms we identified 6,515 potential entities for the vitamin A metabolism pathway. For clarity, we distinguish single-word terms from multiple-word terms. Single-word terms are more likely to be ambiguous than multiword terms. The first assessment of the created terminology was then performed on the multiword terms.

\section{Presenting potential pathway entities in context}

We first presented a list of found terms that could be a potential pathway entity, in a html page (see http://www.bigcat .unimaas.nl/public/data/textmining/carotenoids), where a link to the abstracts from the SEC that contained this term is also presented. The list of abstracts, when opened, shows the sentences containing the concept plus a clickable PubMed identifier to the original publication. This part is primarily meant to verify the textmining results themselves.

To facilitate the contextual evaluation we integrated our newly found entities as a separate list in the carotenoid pathway that is available on WikiPathways.

This allows the curators to use the pathway editors functionality on WikiPathways to review the results, to connect the new entities to the existing pathway, and to add database information to individual concepts, to leave them as a separate list of related entities or to remove them. In general, this mechanism, allows us to integrate results from automated text mining with manual curation.

The final result of our text-mining work flow is a list of terms representing potential pathway entities, which are hyperlinked to their original context of occurrence in the expanded corpus. We have built an interface to browse the terminology (Fig. 5). In this interface the results can be sorted by the JD values and their frequencies. It is also possible to filter for specific semantic types such as enzymes, metabolites, or other chemical compounds.

The terms in Table 2 were selected from the generated vocabulary. They comprised the most informative term, that is, most frequent, highest JD and high frequency but low JD. Table 2 contains those terms that were found with the uniprot filter (Proteins), Table 3 represents the metabolites found with the HMDB filter, and Table 4 contains terms that were

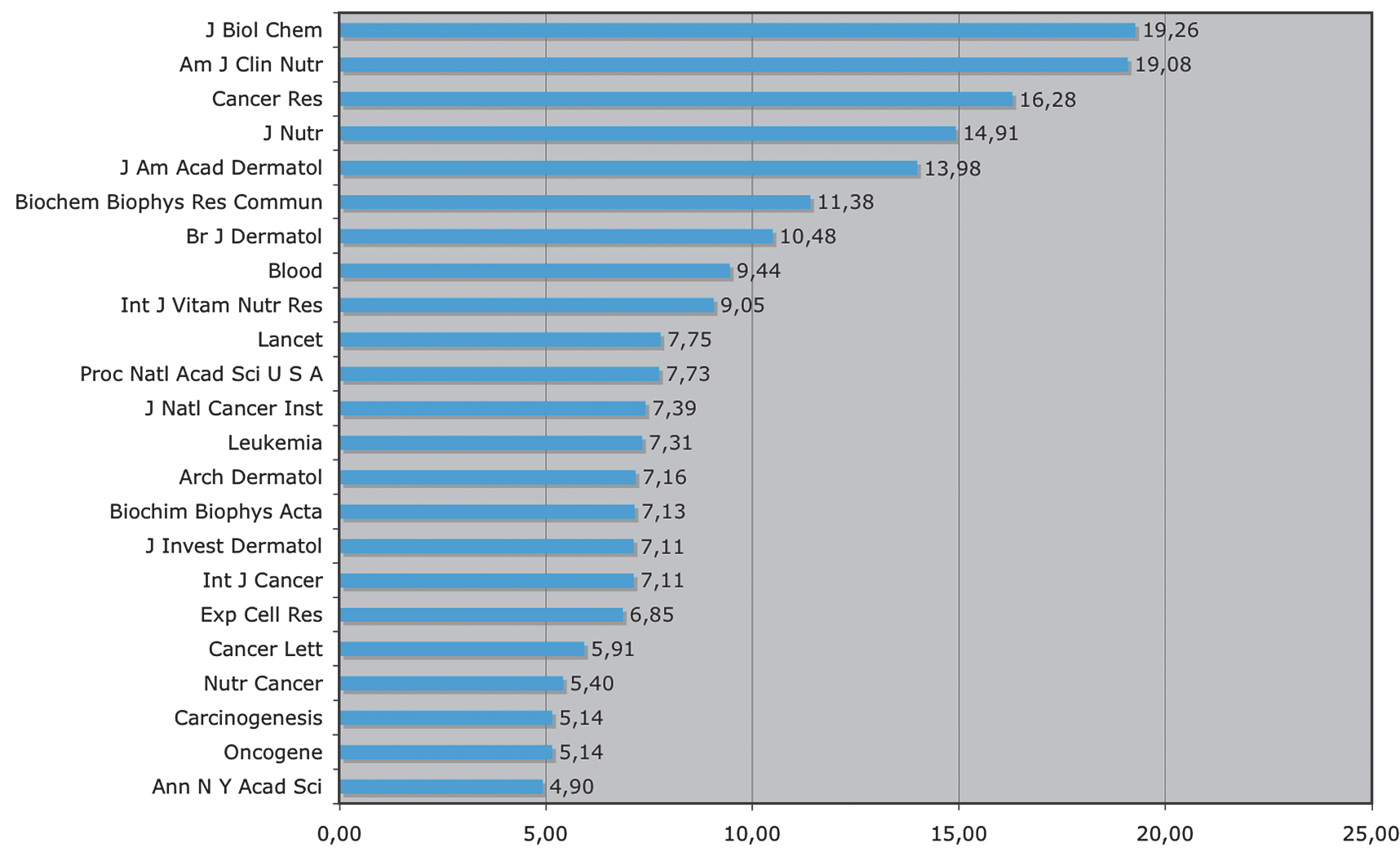

FIG. 4. The journals that had the relative highest increase in contribution to the extended corpus. The increase is measured in $z$-scores. 


\begin{tabular}{|l|l|}
\hline Upload a pathway (gpml) & \\
\hline Corpus & Carotenoids : \\
\hline Number of records & 30 \\
\hline$\odot$ Multiple word & $\bigcirc$ single word \\
\hline Semantic Type & all \\
\hline Sort by: & Jo dispersion \\
\hline JD $<$ 80 & a $>30$ \\
\hline \multicolumn{1}{c|}{ Submit Quern } \\
\hline
\end{tabular}

\begin{tabular}{|c|c|c|c|c|c|c|c|}
\hline & \multicolumn{3}{|c|}{ Number of records: 30} & \multicolumn{4}{|c|}{ (Submit Query } \\
\hline Concept & Context & Uniprot-filter & Chemicals filter & WD & a & Annotation & Comments \\
\hline vitamins $A$ & - $\underline{\text { SEC }}$ & & Metabolite (hmdb) & 95.052 & 3765 & Synonym & vitamin $\mathrm{A}=$ retinol \\
\hline liquid chromatography & $: \frac{\text { SEC }}{\text { MedEvi }}$ & & other chemical or false + & 94.595 & 715 & Irrelevant & $\begin{array}{l}\text { method of analysis of } \\
\text { carotenoids and } \\
\text { retinoids }\end{array}$ \\
\hline fatty acids & $: \frac{\mathrm{SEC}}{\mathrm{MedEvi}}$ & & CHEBI:35366 & 94.05 & 765 & $\div$ & \\
\hline retinoic acids & $: \underline{\text { SedEV }}$ & & Metabolite (hmdb) & 93.697 & 6516 & Synonym & $\begin{array}{l}\text { retinoic acid is a } \\
\text { metabolite of } \\
\text { retinol, and is the } \\
\text { retion }\end{array}$ \\
\hline high-performance liquid chromatography & $: \frac{\mathrm{SEC}}{\mathrm{MedEvi}}$ & & other chemical or false + & +93.679 & 407 & Irrelevant & $\begin{array}{l}\text { Tethod of analysis of } \\
\text { carotenoids and } \\
\text { retinoids }\end{array}$ \\
\hline ascorbic acid & - $\frac{\text { SEC }}{\text { MedEvi }}$ & & Metabolite (hmdb) & 92.624 & 819 & Not Sure & $\begin{array}{l}\text { chemical name of } \\
\text { vitamin c, } \\
\text { hydrophilic }\end{array}$ \\
\hline
\end{tabular}

FIG. 5. The extracted terms from the similarity extended corpus is available to pathway curators (http://www .bigcat.unimaas.nl/public/data/textmining/carotenoids/). Through this interface one could browse the results by sorting on the frequency of occurrence or the Julliand Dispersion (JD). Furthermore, curators could limit the terms on specific semantic types (i.e., proteins, metabolites, chemical compounds). Curators could also consult the context either by extracting the keywords in conjunction with the sentence where they were extracted from, or by using EBI's MedEvi (http://www.ebi .ac.uk/Rebholz-srv/MedEvi/).

identified with the chemical compound filter of Whatizit. These terms were then validated by two curators who were also involved in the creation of the initial pathway (Fig. 1). We provided these terms together with an annotation tool where the curators could classify the potentially new pathway enti- ties according to a predefined set of classes: (1) already included, (2) hyponym of a term already included, (3) hypernym of a term already included, (4) synonym of a term already included, (5) too generic, (6) irrelevant to the studied pathway, and (7) potential new pathway concept.

Table 2. Proteins That Are Potential Pathway Entities in Vitamin A Metabolism

\begin{tabular}{|c|c|c|c|}
\hline \multicolumn{3}{|c|}{ Multiword Uniprot entities } & \multirow[b]{2}{*}{ Single-word Uniprot entities } \\
\hline Sorted on Julliands Dispersion (JD) & Sorted on frequency of occurrence (a) & $J D<70 a>10$ & \\
\hline alkaline phosphatase & protein kinases & tissue-type & dehydrogenases \\
\hline cytochromes P450 & alkaline phosphatase & plasminogen activator & dehydrogenase \\
\hline protein kinases & cytochromes $\mathrm{P} 450$ & Phospholipase A2 & transglutaminase \\
\hline Lipoprotein lipase & Lipoprotein lipase & zeta-carotene & Catalase \\
\hline prostate-specific antigen & prostate-specific antigen & desaturase & peroxidase \\
\hline zeta-carotene desaturase & tissue-type plasminogen activator & triglyceride lipase & GNMT \\
\hline gamma-glutamyl transpeptidase & Phospholipase A2 & gamma-glutamyl & thrombin \\
\hline Phospholipase A2 & zeta-carotene desaturase & transpeptidase & $\mathrm{ACE}$ \\
\hline acid phosphatase & triglyceride lipase & acid phosphatase & TPA \\
\hline cyclin-dependent kinase & gamma-glutamyl transpeptidase & & NOS \\
\hline proteinase $\mathrm{K}$ & acid phosphatase & & AOX \\
\hline DNA methyltransferase & aryl hydrocarbon hydroxylase & & RetSat \\
\hline tissue-type plasminogen activator & DNA methyltransferase & & synthetase \\
\hline Cytochrome oxidase & cyclin-dependent kinase & & $\mathrm{ADH} 3$ \\
\hline triglyceride lipase & MAP kinases & & PEPCK \\
\hline aryl hydrocarbon hydroxylase & proteinase $\mathrm{K}$ & & trypsin \\
\hline MAP kinases & Reverse transcriptase & & Lipase \\
\hline tissue plasminogen activator & tissue plasminogen activator & & ceruloplasmin \\
\hline aldose reductase & aldose reductase & & lysozyme \\
\hline glutamic-oxaloacetic transaminase & Cytochrome oxidase & & TGase \\
\hline Reverse transcriptase & glutamic-oxaloacetic transaminas & & PKA \\
\hline
\end{tabular}


Table 3. Metabolites That Are Potential Pathway Entities in Vitamin A Metabolism

\begin{tabular}{|c|c|c|c|}
\hline \multicolumn{3}{|c|}{ Multiword metabolites } & \multirow[b]{2}{*}{ Single-word metabolites } \\
\hline Sorted on Julliands Dispersion (JD) & Sorted on frequency of occurence (a) & $J D<70 a>10$ & \\
\hline vitamins A & retinoic acids & 25-hydroxyvitamin D & Retinol \\
\hline retinoic acids & vitamins $\mathrm{A}$ & vitamin D2 & beta-carotene \\
\hline ascorbic acid & Vitamin $C$ & phytanic acid & lycopene \\
\hline Retinyl palmitate & retinyl ester & lutein esters & lutein \\
\hline Vitamin C & Retinyl palmitate & nicotinic acid & alpha-tocopherol \\
\hline retinoic acid & ascorbic acid & L-ascorbic acid & zeaxanthin \\
\hline all-trans retinoic acids & all-trans-retinoic acids & 11 -cis retinaldehyde & palmitate \\
\hline Folic acid & retinoic acid & pantothenic acid & alpha-carotene \\
\hline all-trans retinol & retinoic acid & ferulic acids & beta-cryptoxanthin \\
\hline 13-cis-retinoic acid & 13-cis-retinoic acid & copper ion & astaxanthin \\
\hline retinyl ester & beta carotenes & alpha carotene & canthaxanthin \\
\hline all-trans retinoic acid & 9-cis-retinoic acids & adenosine & retinal \\
\hline beta carotenes & Folic acid & monophosphate & Selenium \\
\hline Linoleic acid & Vitamin D3 & chlorogenic acid & Tocopherols \\
\hline Vitamin D3 & all-trans retinol & retinoyl glucuronide & Lycopene \\
\hline beta carotenes & Linoleic acid & isopentenyl & tocopherol \\
\hline 9-cis-retinoic acids & retinol palmitate & diphosphate & phytoene \\
\hline reduced glutathione & all-trans retinoic acid & ethanol solution & zinc \\
\hline Uric acid & Uric acid & glutathione disulfide & cholesterol \\
\hline retinol palmitate & Vitamin $\mathrm{K}$ & caffeic acids & all-trans-retinol \\
\hline oleic acids & Alpha tocopherol & carnosic acid & retinaldehyde \\
\hline nitric oxides & all-trans retinoic acid & retinyl ester & gamma-tocopherol \\
\hline arachidonic acids & beta-carotenes & carbon dioxide & Lutein \\
\hline all-trans retinoic acid & reduced glutathione & cholesterol sulfate & isotretinoin \\
\hline docosahexaenoic acids & all-trans beta-carotene & nitrogen dioxide & iron \\
\hline alpha-tocopherol & arachidonic acids & isopentenyl & cryptoxanthin \\
\hline Superoxide anion & nitric oxides & pyrophosphate & lutein \\
\hline Vitamin K & docosahexaenoic acids & alpha-linolenic acid & glutathione \\
\hline all-trans beta-carotene & $\begin{array}{l}\text { oleic acids } \\
\text { benzoic acids }\end{array}$ & 13-cis retinoic acids & violaxanthin \\
\hline
\end{tabular}

Hypernyms are terms denoting more general entities than the term in question, and conversely, hyponyms are terms that are more specific than a given term. For example, lutein is the name of a carotenoid, that is, lutein is the hyponym of a carotenoid and carotenoid is the hypernym of Lutein.

Our initial validation of the 266 potential pathway entities represented in Tables 2, 3 and 4 reduced the list to 36 potential pathway entities for the vitamin A metabolism pathway (Table 5). During this validation the curators not only read the terms themselves, but also the sentences and abstracts in which these potential pathway entities originally occurred. This validation step, based on the text from the Medline abstracts, also resulted in the identification of yet one additional pathway concept (Vitamin D3 receptor) (Heber and Lu, 2002; Torma et al., 1996; Vasarhelyi et al., 1993), which had not been identified by the automatic method, but has been identified by reading the most relevant abstracts. This concept has not been identified automatically because it represents a more complex multiword concept just like vitamin D3 receptor. Although the bigram vitamin D3 was identified by the automatic approach the trigram was not. Taking this additional concept into consideration we had a total of 37 pathway entities that have been identified as result of our semiautomatic extraction methods.

In the next step, the domain experts tried to incorporate the novel entities into the pathway. This processing step led to the result that 12 of the 36 terms and, in addition, the term vitamin
D3 receptor (the 37th term) were found to be pathway entities that had to be included in the pathway for vitamin A metabolism. Fourteen of the remaining 24 entities were attributed to processes of related metabolic pathways like fatty acid metabolism, oxidative stress, coagulation cascade, and cholesterol metabolism. Because such entities form possibly yet unknown links to related pathways, we suggest that text mining not only serves finding new pathway entities but could also have a role in identifying related pathways.

The 10 remaining terms could be categorized either as nutrients or biomarkers. Vitamin A, carotenoids and other pathway concepts are frequently used as biomarkers in clinical and other health studies.

The pathway containing the 13 new pathway entities was given to the expert team on carotenoid metabolism (NuGO focus team). They judged on the relevance of the modifications to the pathway representation and considered the additions as a relevant improvement (Fig. 6).

\section{Discussion}

Identification of pathways is ongoing research work. Extraction of pathway-relevant information from the literature, including protein-protein interactions and molecular interactions, is a challenging task. In the current state, curation of involved entities and integration of novel entities into existing pathways is necessary to deliver high-quality resources to the 
Table 4. Chemical Compounds That Are Potential Pathway Entities in Vitamin A Metabolism

Multiword chemicals

\begin{tabular}{|c|c|c|c|}
\hline Sorted on Julliands Dispersion (JD) & Sorted on frequency of occurence (a) & $J D<70 a>10$ & Single-word chemicals \\
\hline liquid chromatography & fatty acids & arsenic trioxide & carotenoids \\
\hline fatty acids & liquid chromatography & phenolic acid & retinoic \\
\hline high-performance liquid & retinyl acetates & retinyl phosphate & carotenoid \\
\hline chromatography & high-performance liquid & unesterified retinol & retinyl \\
\hline polyunsaturated fatty acids & chromatography & Raman spectroscopy & retinoids \\
\hline fatty acids & amino acid & all-rac-alpha-tocopheryl acetate & retinoid \\
\hline retinyl acetates & hepatic vitamin & Stanol ester & acid \\
\hline In spite & polyunsaturated fatty acids & lipoprotein cholesterol & antioxidant \\
\hline amino acid & retinol acetate & CRBP I & carotene \\
\hline electron microscopy & double bond & 25-dihydroxyvitamin D & retinoic \\
\hline high-pressure liquid & fatty acids & acyl coenzyme & all-trans-retinoic \\
\hline chromatography & vitamins B6 & heme protein & esters \\
\hline thiobarbituric acid & high-pressure liquid & tannic acid & lipid \\
\hline Hepatic vitamin & chromatography & retinyl methyl ether & peroxidation \\
\hline hydrogen peroxide & phorbol esters & phosphatidyl choline & nutrients \\
\hline vitamins B6 & cholesteryl ester & petroleum ether & 9-cis \\
\hline free radical & bile acids & lipoprotein oxidation & provitamin \\
\hline double bond & energy transfer & mitomycin C & pigment \\
\hline gel electrophoresis & radical cations & cytosine arabinoside & ascorbic \\
\hline Retinol acetate & electron microscopy & cyclin E & $13-c i s$ \\
\hline cholesteryl ester & In spite & Eimeria acervulina & micronutrient \\
\hline Subclinical vitamin & hydrogen peroxide & vitamin $\mathrm{K} 1$ & retinoids \\
\hline chain reaction & free radical & ethyl acetate & 13-cis-retinoic \\
\hline Carotenoids Lutein & photosystem II & redox state & pigments \\
\hline Gel filtration & thiobarbituric acid & radical anion & acid \\
\hline Messenger RNA & Retinol oxidation & 13-cis-4-oxoretinoic acid & oxidation \\
\hline vitamin B & gel filtration & Retinyl beta-glucuronide & 9-cis-retinoic \\
\hline gas chromatography & bile salt & 11-cis-retinyl palmitate & 9-cis \\
\hline Actinomycin D & retinyl stearate & 6-epoxyretinoic acid & Retinoids \\
\hline steroid hormone & collagen synthesis & Mannosyl retinyl phosphate & xanthophylls \\
\hline liquid chromatography & chain reaction & & \\
\hline Phorbol esters & subclinical vitamin & & \\
\hline
\end{tabular}

public. In our study, we have semiautomatically produced a list of entities that were finally included into the representation of the carotenoid pathways. Other approaches (Spasic et al., 2008) have tackled the same problem but they have not been evaluated for the integration of novel entities into existing pathways. Nonetheless, both approaches demonstrate that the scientific literature is still a very rich resource to gather relevant information for the completion of pathway representations.

One crucial step in our analysis is the generation of a corpus that is expanded from the SC and that can be used as input to

Table 5. Of the Retrieved terms, Which Were Assessed as Being Relevant to the Vitamin A Pathway, 12 Proved to be Pathway Entities, 13 Are Entities in Related Pathways and 10 Are Not Related But Were Identified, Because They Are Administered or Used as Biomarkers in Clinical Studies

\begin{tabular}{lll}
\hline New pathway entities & \multicolumn{1}{c}{ Related processes or pathways } & Biomarkers or nutrients \\
\hline Vitamin D3 & linoleic acid & selenium \\
ADH3 & fatty acid & reduced glutathione gamma-tocopherol \\
alpha-carotene & GNMT & alpha-tocopherol \\
astaxantin & MAPK & zinc \\
beta-cryptoxanthin & NOS & vitamine B \\
Canthaxanthin & nitric oxides & vitamin K \\
lutein & oleic acids & vitamine B6 prostate-specific antigen \\
lycopene & TGase & \\
zeaxanthin & carboxylesterases & \\
PEPCK & cholesterol & \\
cryptoxanthin & arachidonic acids & \\
triglyceride lipase & docosahexaenoic acids & \\
& tissue-type plasminogen activator & \\
& aldose reductase & \\
\hline
\end{tabular}




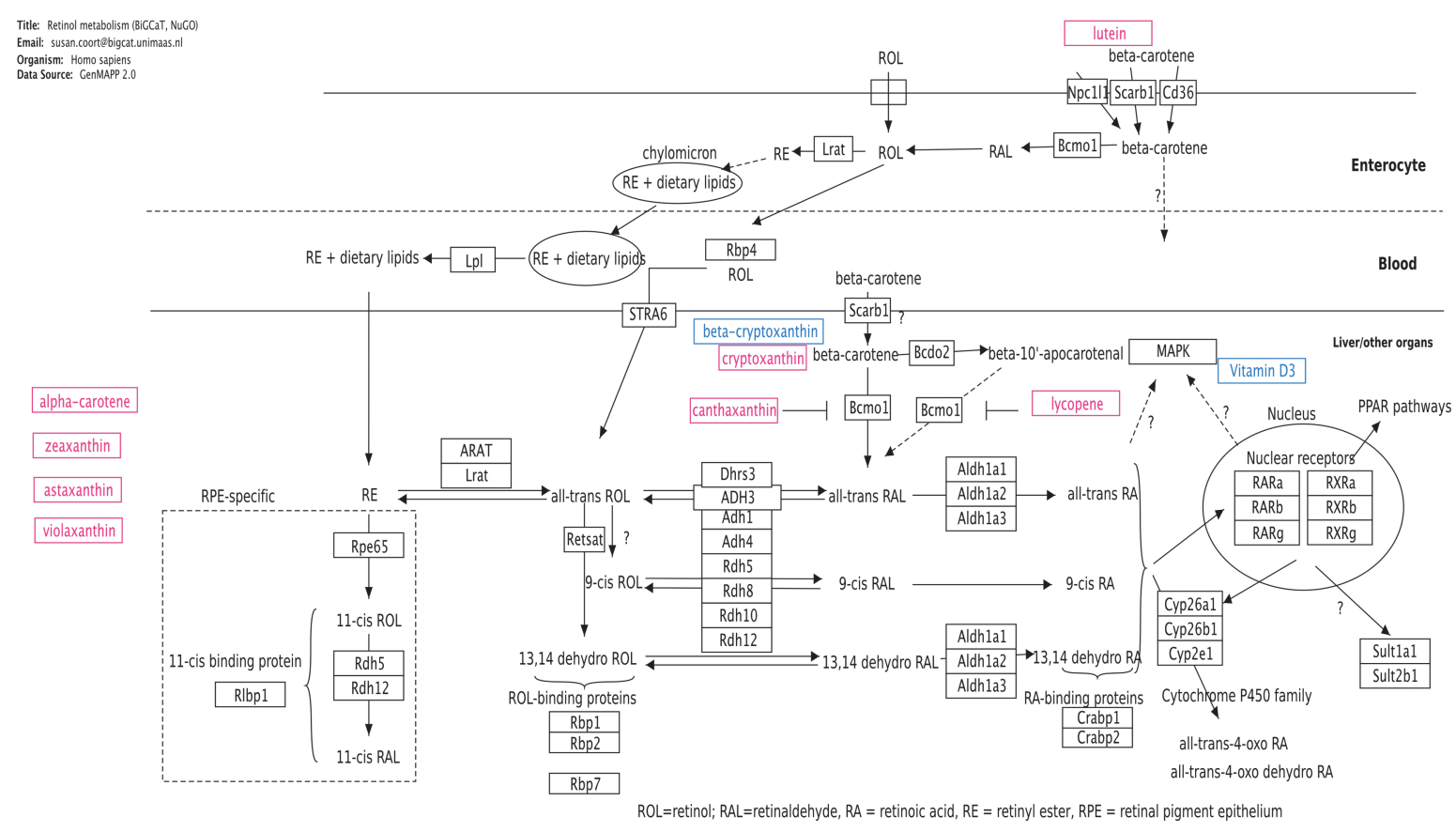

FIG. 6. The resulting pathway of vitamin A metabolism extended with a text-mining approach. The newly included entities are indicated with colored boxes.

the extraction of the key terminology. In our study we have a review containing 1,191 references, which forms an excellent basis for a SC. Often such reviews are not available. In that case, the SC needs to be compiled by consulting experts. The resulting start set can in some cases be extended with references that were added to the studied pathway itself. KEGG and WikiPathways contain such references. This start set can in fact contain reviews like the one we used, which then can, of course, be extended. In other cases Web resources like CiteULike (http://www.citeulike.org/) and Connotea (http:// www.connotea.org/), which provide means for scientists to organize and share references, can be used to find other articles that were often saved in combination with the articles from the start set.

The selection of the extended corpus was based on a scoring function that discriminates between suitable documents and irrelevant documents with an empirically defined cutoff parameter. This distinction is arbitrary to a given extent, but ensures that the best-ranked documents according to the judgment of a domain expert are included in the study.

The main analysis is concerned with the selection of terminology and the assessment of this terminology by domain experts. The overall number of identified entities is small (only 37) in comparison to the overall number of extracted terms $(89,086$ terms). This reduction step is crucial to the selection of the most relevant terms and to optimize the interaction between the text-mining research group and the team of domain specialist, that is, the amount of relevant terms has to be small enough to be processed by the domain specialists and comprehensive enough to generate sufficient benefits from their work.

The identification of novel terminology from the literature for the completion of pathway representations still requires human assessment to meet the high-quality standards of $\mathrm{cu}$ rated data resources. This will not change in the near future.
On the other side, our study has shown that it is beneficial to consider the whole of Medline for an analysis to search for a small number of relevant terms. It is clear that the initial set of documents that have been selected from a review article did not make reference to all journals that could be relevant to the identification of entities for the selected pathway. In other words, only a text-mining approach that filters information from a large set of documents does not have to rely on assumptions that make a preselection of documents, journals, authors, or keyword queries. Considering the whole of the scientific literature gives advantages in the sense that no restrictions are applied to the primary data resource, and thus one of the many reasons for a bias to the study's results is excluded.

For the future we expect that other research teams will pick up similar approaches to filter the literature for concepts that have to be included into biomedical data resources and ontology's. To some extent, this need will be answered by online search engines that offer efficient access to the scientific literature (Doms and Schroeder, 2005; Kim et al., 2008). In addition, researchers will have to work together with text-mining research teams to identify the most relevant concepts. Altogether, the literature will be filtered many times for different purposes and will disclose step-by-step pieces of information that will be integrated into the public biomedical data resources.

\section{Conclusions and Future Work}

The increase in the volume of scientific literature requires new methods for knowledge extraction from the scientific literature. Our approach focused on the carotenoid pathway (retinol metabolism). We generated an extended corpus of Medline abstracts and based on the evaluation of sample sets, we conclude that the final extended corpora contained 
additional pathway relevant documents that could only be gathered in a time-consuming manual process otherwise. With the combination of the corpus expansion and the pathway enrichment work flow, we were able to identify 13 relevant and yet uncovered entities that were included into the vitamin A metabolism pathway, which is part of the carotenoid pathway. Our results also show that concept can be identified that are potential links with other processes or pathways. The expanded corpus and the extracted terminology including links to relevant Medline abstracts are available at http://www.bigcat.unimaas.nl/public/data/textmining/ carotenoids/

The work flow is applicable on other domains. We have also applied it on the extraction of pathways entities of the selenium metabolism pathway. Curators are currently processing the results into extending the existing pathway diagram.

The evaluation of the results of our work flow still requires significant human intervention. With this work flow we were able to select the most important information from much more abstracts than a given number of domain experts would be able to read, interpret, and understand. Essentially, we took the information from 13,579 abstracts preselected both by direct queries and by similarity evaluation with a seed corpus. Moreover, the application of the JD value helps in finding rare entities that are only described in a small part of the selected abstracts. The chance of missing these entities in a manual literature search is high.

We believe that the human intervention is decreased if our work flow is integrated in pathway repositories such as WikiPathways (Pico Ar, 2008). The community then provides the seed corpora. The final result of the work flow is a terminology of potential pathway entities. This could be provided as a list with references to the literature, so that the curators (i.e., the community) can make a well-considered decision on extending the different pathways. To allow integration of this work flow in WikiPathways, we extended this wiki with an interface for automatically generated suggestions.

The pathway is available on the Web page of WikiPathways (Pico Ar, 2008) (http://www.wikipathways.org) for download. It can be used for gene expression analyses in Genmapp (Dahlquist et al., 2002) and EU Gene (Cavalieri et al., 2007) and representations of interactions in Cytoscape (Shannon et al., 2003).

\section{Authors' Contributions}

A.W., P.P., and D.R.S. conceived and implemented the textmining work flow. S.C., F.T., and C.E. analyzed the result and provided the expert knowledge for the validation of results. All authors provided content for the final manuscript.

\section{Acknowledgments}

This research has been made possible by the close cooperation of five research institutes. We are grateful to the management of these institutes who enabled the researchers to travel and visit each other. In particular, we would like to mention the Netherlands Bioinformatics Community for their support via Biorange Phasar project (SP 4.1.1). Furthermore, we are grateful to the Netherlands Genomics Initiative for their financial support to make Andra Waagmeester 6-month fellowship and collaborate with the European Bioinformatics Institute in Cambridge (UK). We are also grateful to the European Network of Excellence in Nutrigenomics (NuGO) and its carotenoid focus team for providing a framework from wherein researchers from different scientific domains are able to collaborate.

\section{Author Disclosure Statement}

The authors declare that no conflicting financial interests exist.

\section{References}

Amiot-Carlin, M.J. (2008). Plant pigments as bioactive substances. In: Food Colorants: Chemical and Functional Properties. (CRC Press, Boca Raton, FL), pp. 127-146.

Bader, G.D., Cary, M.P., and Sander, C. (2006). Pathguide: a pathway resource list. Nucleic Acids Res 34, D504-D506.

Bairoch, A., Apweiler, R., Wu, C.H., Barker, W.C., Boeckmann, B., Ferro, S., et al. (2005). The Universal Protein Resource (UniProt). Nucleic Acids Res 33, D154-D159.

Balmer, J.E., and Blomhoff, R.(2002). Gene expression regulation by retinoic acid. J Lipid Res 43, 1773-1808.

Bendich, A., and Olson, J.A. (1989). Biological actions of carotenoids. FASEB J 3, 1927-1932.

Cary, M., Bader, G., and Sander, C. (2005). Pathway information for systems biology. FEBS Lett 579, 1815-1820.

Cavalieri, D., Castagnini, C., Toti, S., Maciag, K., Kelder, T. Gambineri, L., et al. (2007). Eu.Gene Analyzer a tool for integrating gene expression data with pathway databases. Bioinformatics 23, 2631-2632.

Chen, H., and Sharp, B.M. (2004). Content-rich biological network constructed by mining PubMed abstracts. BMC Bioinformatics 5, 147.

Dahlquist, K.D., Salomonis, N., Vranizan, K., Lawlor, S.C., and Conklin, B.R. (2002). GenMAPP, a new tool for viewing and analyzing microarray data on biological pathways. Nat Genet 31, 19-20.

Degtyarenko, K., De Matos, P., Ennis, M., Hastings, J., Zbinden, M., McNaught, A., et al. (2008). ChEBI: a database and ontology for chemical entities of biological interest. Nucleic Acids Res 36, D344-D350.

Doms, A., and Schroeder, M. (2005) GoPubMed: exploring PubMed with the Gene Ontology. Nucleic Acids Res 33, W783-W786.

Harrison, E.H. (2005). Mechanisms of digestion and absorption of dietary vitamin A. Annu Rev Nutr 25, 87-103.

Heber, D., and Lu, Q.-Y. (2002). Overview of mechanisms of action of lycopene. Exp Biol Med (Maywood) 227, 920-923.

Jensen, L.J., Saric, J., and Bork, P. (2006). Literature mining for the biologist: from information retrieval to biological discovery. Nat Rev Genet 7, 119-129.

Kim, J.J., Pezik, P., and Rebholz-Schuhmann, D. (2008). MedEvi: retrieving textual evidence of relations between biomedical concepts from Medline. Bioinformatics 24, 1410-1412.

Kirsch, H., Gaudan, S., and Rebholz-Schuhmann, D. (2006). Distributed modules for text annotation and IE applied to the biomedical domain. Int J Med inform 75, 496-500.

Krinsky, N.I. (1993). Actions of carotenoids in biological systems. Annu Rev Nutr 13, 561-587.

Oakes, M.P. (1998). Statistics for Corpus Linguistics. Edinburgh: Edinburgh Textbooks in Empirical Linguistics, Edinburgh University Press. 
Pico Ar, K.T., Van Iersel, M.P., Hanspers, K., Conklin, B.R., and Evelo, C.T. (2008). WikiPathways: Pathway Editing for the People. PLOS Biol.

Rebholz-Schuhmann, D., Arregui, M., Gaudan, S., Kirsch, H., and Jimeno, A. (2008). Text processing through Web services: calling Whatizit. Bioinformatics 24, 296-298.

Shannon, P., Markiel, A., Ozier, O., Baliga, N.S., Wang, J.T., Ramage, D., et al. (2003). Cytoscape: a software environment for integrated models of biomolecular interaction networks. Genome Res 13, 2498-2504.

Shatkay, H., and Feldman, R. (2003). Mining the biomedical literature in the genomic era: an overview. J Comput Biol 10, 821-855.

Spasic, I., Schober, D., Sansone, S.A., Rebholz-Schuhmann, D., Kell, D.B., and Paton, N.W. (2008). Facilitating the development of controlled vocabularies for metabolomics technologies with text mining. BMC Bioinformatics 9(Suppl 5), S5.

TREC. (2007). The Sixteenth Text Retrieval Conference Proceedings.

Torma, H., Rollman, O., Binderup, L., and Michaelsson, G. (1996). Vitamin D analogs affect the uptake and metabolism of retinol by human epidermal keratinocytes in culture. J Invest Dermatol Symp Proc 1, 49-53.
Van Ommen, B., Fairweather-Tait, S., Freidig, A., Kardinaal, A., Scalbert, A., and Wopereis, S. (2008). A network biology model of micronutrient related health. Br J Nutr 99(Suppl 3), S72-S80.

Vasarhelyi, B., Blazovics, A., and Feher, J. (1993). [The role of vitamin A analogues and derivatives in the regulation of cell function]. Orv Hetil 134, 845-848.

Voutilainen, S., Nurmi, T., Mursu, J., and Rissanen, T.H. (2006). Carotenoids and cardiovascular health. Am J Clin Nutr 83, 1265-1271.

Wishart, D.S., Tzur, D., Knox, C., Eisner, R., Guo, A.C., Young, N., et al. (2007). HMDB: the Human Metabolome Database. Nucleic Acids Res 35, D521-D526.

Yeum, K.J., and Russell, R.M. (2002). Carotenoid bioavailability and bioconversion. Annu Rev Nutr 22, 483-504.

Address correspondence to: Andra Waagmeester Department of Informatics-BiGCaT Maastricht University P.O. Box 616 6200 MD Maastricht, The Netherlands E-mail: andra.waagmeester@bigcat.unimaas.nl 
